\title{
New Drosophila transgenic reporters: insulated P-element vectors expressing fast-maturing RFP
}

\author{
Scott Barolo, Brian Castro, and James W. Posakony \\ University of California San Diego, La Jolla, CA, USA
}

BioTechniques 36:436-442 (March 2004)

In vivo green fluorescent protein (GFP)/red fluorescent protein $(R F P)$ double-labeling studies have been hampered by several inconvenient properties of DsRed, the first described RFP. These disadvantages include a very slow (>24 h) maturation time, emission of contaminating green light, and low solubility. A recently developed variant of DsRed, called DsRed.T4, has a much shorter maturation time, no significant green emission, and improved solubility. We have constructed Drosophila P-element transformation vectors encoding DsRed.T4 for promoter/enhancer analysis, labeling of living cells, or RFP tagging of proteins. These new vectors have all of the features of the widely used Pelican/Stinger GFP vectors, including insulator sequences to reduce position effects, an extensive polylinker, and both cytoplasmic and nuclear-localized forms of the reporter. We have also constructed an upstream activating sequence (UAS)-DsRed.T4 vector, for GAL4 activation of the reporter. We find that DsRed.T4 is very easily detected in transgenic flies without contamination of the GFP signal and that it matures to its fluorescent form nearly simultaneously with GFP. This advance in Drosophila reporter technology makes timed double-labeling experiments in developing transgenic animals possible for the first time.

\section{INTRODUCTION}

Green fluorescent protein (GFP), which can be used to label living organisms and cells and can be expressed in cell type-specific patterns, has quickly become an indispensable tool for researchers in all fields of molecular biology. Since the ascendance of GFP, many researchers have grasped the potential applications of a red fluorescent protein (RFP) with which one could fluorescently label two cell types in a single living organism or two proteins in a living cell. Recently, an RFP from the coral Discosoma has been described (1-4). This protein, called DsRed, is related to GFP and, like GFP, can be excited by a 488-nm argon laser but has an emission maximum of $583 \mathrm{~nm}$ (compared to $500 \mathrm{~nm}$ for GFP). However, wild-type DsRed and its codon-optimized variant DsRed1 (BD Biosciences Clontech, Palo Alto, CA, USA) have not proved useful for developmental two-color studies for two main reasons. First, and most important, the very long chromophore maturation time (half-time $>24$ h) (5) of DsRed means that gene expression studies often cannot be carried out within a developmentally relevant time frame. Second, during an early step in DsRed chromophore maturation, the protein produces a green fluorescence (5), contaminating the GFP signal in double-label studies. Indeed, in our own experiments, we found that DsRed1 matures far too slowly for developmental studies in transgenic flies (data not shown). Recently, Bevis and Glick (6) reported the creation of a mutant form of DsRed1, called DsRed.T4, with a dramatically accelerated maturation time (half-time $<43 \mathrm{~min}$ ) and no detectable contamination of the GFP signal.

We have constructed Drosophila Pelement transformation vectors encoding DsRed.T4 for promoter/enhancer analysis or for labeling live cells. These vectors have all of the features of our widely used Pelican/Stinger enhanced GFP (EGFP) vectors (7), including "insulator" sequences to reduce position effects, an extensive polylinker, a minimal promoter, and the choice of either cytoplasmic or nuclear-localized forms of the reporter. We have also made an upstream activating sequence (UAS)-DsRed.T4 construct for GAL4 activation of the reporter. We find that DsRed.T4 is very easily detected in transgenic flies without cross-contami- nation of the GFP signal and that its maturation time in transgenic animals is similar to that of EGFP. This advance in reporter technology makes timed live double-labeling experiments possible for the first time, allowing, for example, direct comparisons of wild-type and mutant forms of an enhancer in a single animal, or simultaneous fluorescence-activated cell sorting (FACS) purification of two labeled cell populations from staged live tissue.

\section{MATERIALS AND METHODS}

\section{Vector Construction}

pRed H-Pelican (see Figure 1) was constructed by replacing the nuclear EGFP coding sequence in $\mathrm{pH}-\mathrm{Stinger}$ (7) with a PCR product including the coding sequence of DsRed.T4. pRed $\mathrm{H}$-Stinger (Figure 1) was made by adding a $B s r$ GI fragment containing codons 84-122 of the transformer gene, encoding a nuclear localization signal (NLS) (8), to the C-terminal end of the DsRed.T4 coding sequence at a BsrGI site created by PCR (DsRed.T4NLS). Sequences of both vectors are available in GenBank ${ }^{\circledR}$, accession nos. AY342347 and AY342348, respectively. Both vectors are available from the Drosophila Genomics Resource Center at Indiana University. pUASRed Stinger (GenBank accession no. AY490568) was constructed by replacing the EGFP-NLS coding sequence of pUAS-Stinger $(7,9)$ with the DsRed.T4-NLS coding sequence.

Suppressor of Hairless [Su(H)] autoregulatory socket enhancer (ASE) reporters: a 1.9-kb genomic DNA fragment that includes the ASE of the $S u(H)$ gene (10) was ligated into the multiple cloning sequences (MCS) of pH-Stinger, pRed $\mathrm{H}-\mathrm{Pelican}$, and $\mathrm{pRed} \mathrm{H}-\mathrm{Stinger}$.

Enhancer of split $m \alpha$ [E(spl)m $\alpha]$ reporters: a 1.0-kb genomic DNA fragment from the $E(s p l) m \alpha$ gene (-71 to -1083), flanked by endogenous BamHI and $\mathrm{XhoI}$ restriction sites, was amplified by PCR and cloned into the MCS of pH-Stinger. A PCR fragment containing this same region, but with the BamHI and XhoI sites replaced with EcoRI and BamHI sites, respectively, was cloned into the MCS of pRed H-Stinger. 


\section{Germline Transformation of Drosophila}

$\mathrm{P}$ element-mediated germline transformation of Drosophila was carried out as described by Rubin and Spradling (11), using $w^{1118}$ as the recipient strain.

\section{Sample Preparation}

Abdomens shown in Figure 2A were freshly dissected and unfixed. Nota in Figure 2, B and C, were fixed in $4 \%$ paraformaldehyde in phosphatebuffered saline (PBS) overnight at $4^{\circ} \mathrm{C}$ and mounted in $70 \%$ glycerol. Wing imaginal discs in Figure 3 were fixed in $4 \%$ paraformaldehyde in PBS at room temperature for $30 \mathrm{~min}$ and mounted in $85 \%$ glycerol, $100 \mathrm{mM}$ Tris- $\mathrm{HCl}, \mathrm{pH}$ 8.0, and $2.5 \%$ DABCO.

\section{Confocal Microscopy}

A Leica TCS SP2 microscope (with Leica Confocal Software v2.5; both from Leica Microsystems, Bannockburn, IL, USA) was used for confocal imaging. Images in Figure 2 are maximum projections of stacks of 16 frames (Figure 2A), eight frames (Figure 2B), or six frames (Figure 2C) taken along the apical-basal axis. Images in Figure 3 are averaged projections of stacks of 10 frames taken along the apical-basal axis. For Figure 2, fluorophores were excited at $488 \mathrm{~nm}$, and emissions were collected

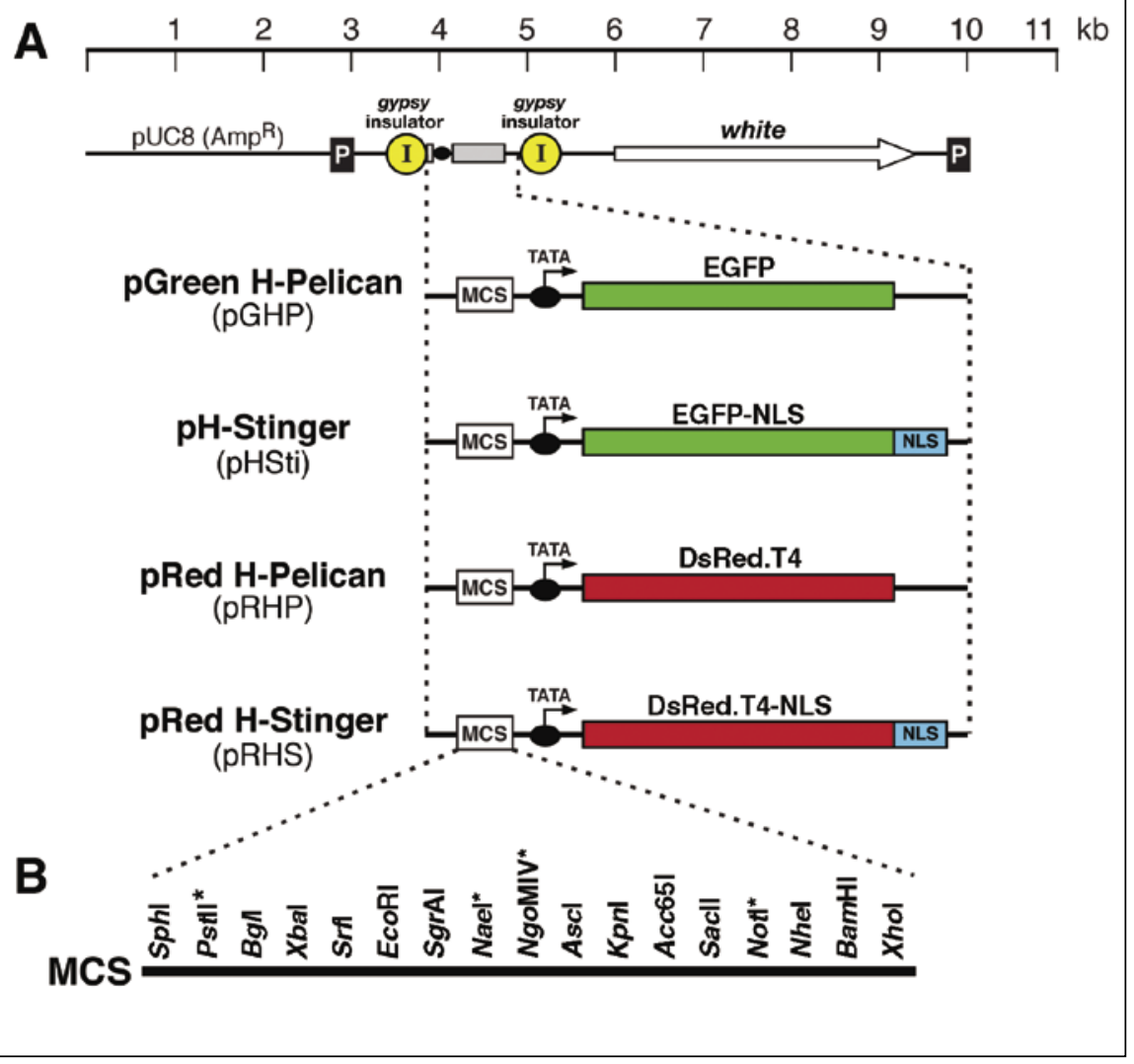

Figure 1. Insulated enhanced green fluorescent protein (EGFP) and red fluorescent protein (RFP) vectors. (A) Diagram of GFP/RFP vectors. All vectors use the mini-white gene as a transformation marker, and all vectors shown contain a minimal Heat shock protein 70 (Hsp70) promoter (TATA, black oval and arrow) driving the reporter gene. Black boxes labeled $\mathrm{P}$ represent terminal P-element sequences required for transposition. Yellow circles labeled I represent transcriptional insulator sequences from the gypsy transposable element. The white rectangle represents the multiple cloning sequence (MCS). Codons for a C-terminal nuclear localization signal (NLS) from the transformer gene are indicated. (B) Unique restriction sites in the MCS of these vectors. Asterisks mark sites that are not unique in all vectors. RFP vector sequences are available in GenBank, accession nos. AY342347 (pRed H-Pelican) and AY342348 (pRed H-Stinger). Complete vector sequences and sample images are available at www.biology.ucsd.edu/labs/posakony. 
at $500-550 \mathrm{~nm}$ (GFP) and $635-710 \mathrm{~nm}$ (DsRed). For Figure 3, fluorophores were excited separately at $488 \mathrm{~nm}$ (GFP) and $543 \mathrm{~nm}$ (DsRed), and emissions were collected at $490-530 \mathrm{~nm}$ (GFP) and 630-710 nm (DsRed).

\section{RESULTS AND DISCUSSION}

We have constructed a series of Drosophila P-element transformation vectors that express a fast-maturing RFP for promoter/enhancer analysis or for marking live cells (Figure 1). pRed H-Pelican encodes DsRed.T4, which localizes to the cytoplasm in vivo, while pRed H-Stinger encodes DsRed.T4-NLS, a nuclear-localized form of the protein (Figure 1A). These new vectors incorporate all of the features of the widely used Pelican/Stinger EGFP vectors (7), including: (i) transcriptional insulator sequences flanking the reporter gene, which reduce "position effects" on gene expression; (ii) a minimal TATA-containing promoter sequence; (iii) an extensive MCS containing 14 to 15 unique restriction sites for the cloning of cisregulatory sequences (Figure 1B); and (iv) a unique restriction site (for SpeI) downstream of the reporter gene, allowing the replacement of the reporter with a gene of choice. A UAS-DsRed.T4NLS construct (pUAS-Red Stinger) is also available for GAL4-driven expression experiments.

To determine whether DsRed.T4 is useful as a counterpart to EGFP in timed developmental studies, we generated transgenic flies that express either the cytoplasmic or nuclear-localized form of the protein, driven by the ASE of the $S u(H)$ gene (10). These flies were then crossed to flies carrying ASE-EGFP-NLS (10). By restricting the collection parameters of the red and green channels, we were able to prevent bleed-through of DsRed.T4 fluorescence into the green channel and of EGFP fluorescence into the red (Figure 2A). Although these settings excluded the majority of the DsRed.T4 signal, the reporter was extremely bright, allowing us to easily collect enough light at the longer wavelengths (Figure 2, A-C).

DsRed.T4 shows a speckled pattern of fluorescence in cells of transgenic Drosophila (Figure 2B). This pattern is consistent with observations of DsRed in other organisms and may be due to protein aggregation, although the $\mathrm{T} 4$ variant reportedly shows less aggregation in vitro than the wild-type protein (6). The nuclear-localized form of the reporter, DsRed.T4-NLS, includes amino acids 84-122 of the transformer gene product (8) fused to the C-terminal end of DsRed.T4; this alteration causes no detectable loss of fluorescence. DsRed.T4-NLS forms speckles within

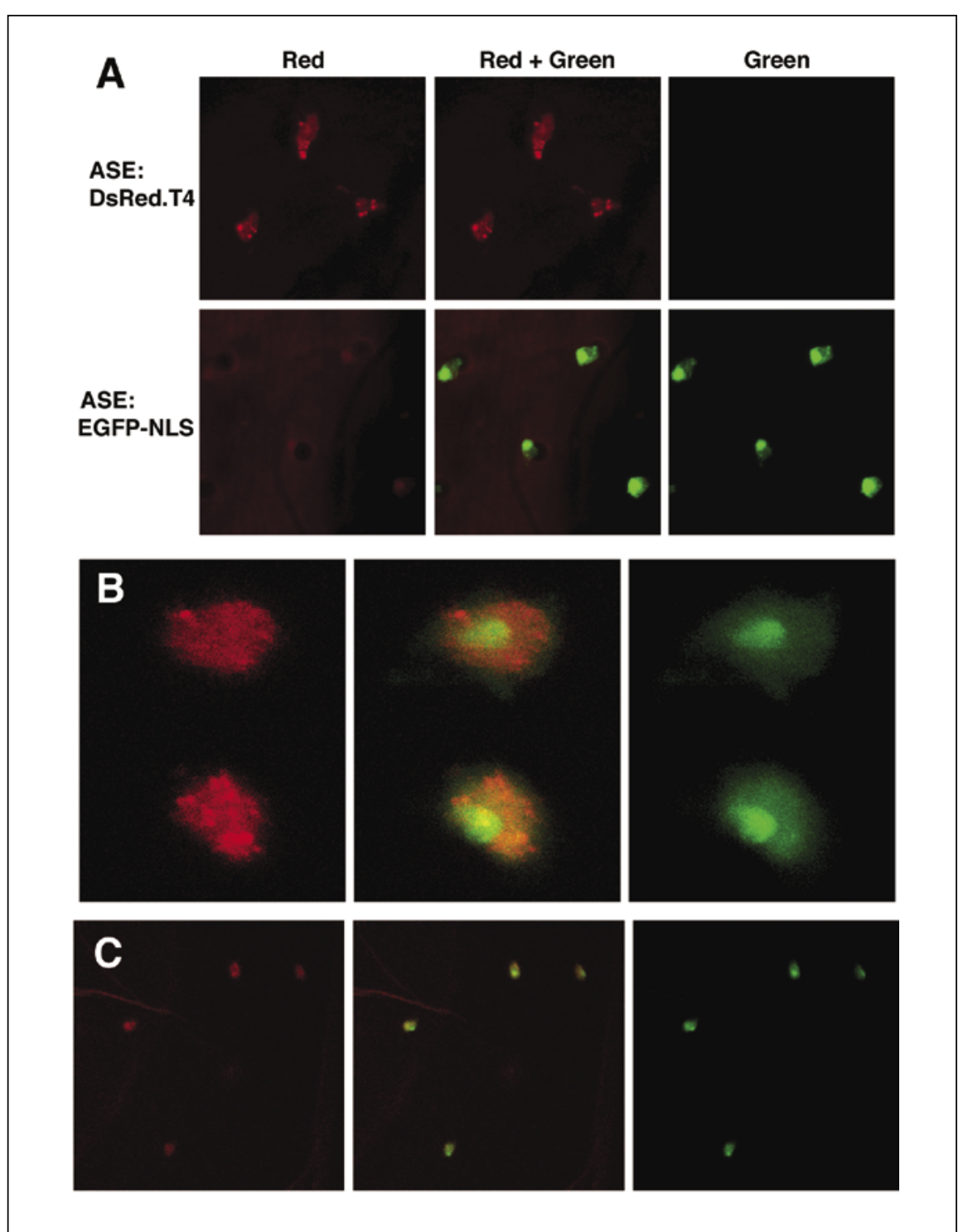

Figure 2. DsRed.T4 and DsRed.T4-NLS in transgenic flies. (A) Fluorescence from DsRed.T4 and enhanced green fluorescent protein (EGFP) can be observed by confocal microscopy with no detectable cross-contamination. (A, top panels) Autoregulatory socket enhancer (ASE) [an enhancer of the Suppressor of Hairless $(\mathrm{Su}[\mathrm{H}])$ gene (10)] driving DsRed.T4 expression in socket cells of adult abdominal bristles. No signal is detected in the green channel (top right panel). (A, bottom panels) ASE driving EGFP-NLS expression in adult abdominal bristles. EGFP is not detected in the red channel, though the cuticle shows some red autofluorescence (bottom left panel). (B) Differing subcellular localization of DsRed.T4-NLS and EGFP-NLS. Shown are socket cells from two macrochaete bristles of a 24-h pupa carrying one copy each of ASE-DsRed.T4-NLS and ASE-EGFP-NLS. (B, left panel) DsRed.T4-NLS labels the nucleus with bright speckles. (B, right panel) EGFP-NLS primarily labels one large subdomain of the nucleus and is expressed at a lower level elsewhere in the nucleus. Fluorescence is also faintly detectable in the cytoplasm. (C) Relative timing of DsRed.T4 and EGFP fluorescent activity during development. Shown is a field of microchaete bristles in the notum of a transgenic 20-h pupa carrying one copy each of ASE-DsRed.T4-NLS and ASE-EGFP-NLS. Not all socket cells are labeled at this stage, but those that are show both red and green fluorescence, indicating that DsRed.T4 and EGFP have similar maturation times under these conditions. NLS, nuclear localization signal. 
the nucleus, with no detectable signal in the cytoplasm (Figure 2B). By contrast, our EGFP-NLS, which contains the same nuclear localization signal (7), does not form speckles, is concentrated in a large subnuclear structure (most likely the nucleolus), and faintly labels the cytoplasm as well (Figure 2B). This weak cytoplasmic signal is not detected with other transgenic constructs (12) and is most likely due to the very strong activity of the ASE (10).

Our greatest concern was whether DsRed.T4 matures quickly enough to provide a feasible counterpart to EGFP in developmental studies, in which timing and developmental stage are important factors in gene expression analysis. By examining nota of transgenic flies carrying both ASE-DsRed.T4-NLS and ASE-EGFP-NLS at a time when this enhancer is first active in socket cells $(20 \mathrm{~h}$ after puparium formation), we could determine the lag time between the appearance of green fluorescence and red fluorescence. Surprisingly, we observed no difference in time-to-detection between the two reporters; green and red fluorescence appeared simultaneously in these cells, and no cells were observed to have only green (or only red) fluorescence (Figure 2C). DsRed.T4 can therefore be used along with GFP in double-label experiments to test the relative timing of expression of two enhancers in the same live transgenic animal.

It should be noted that, while ASEDsRed.T4 flies develop normally and

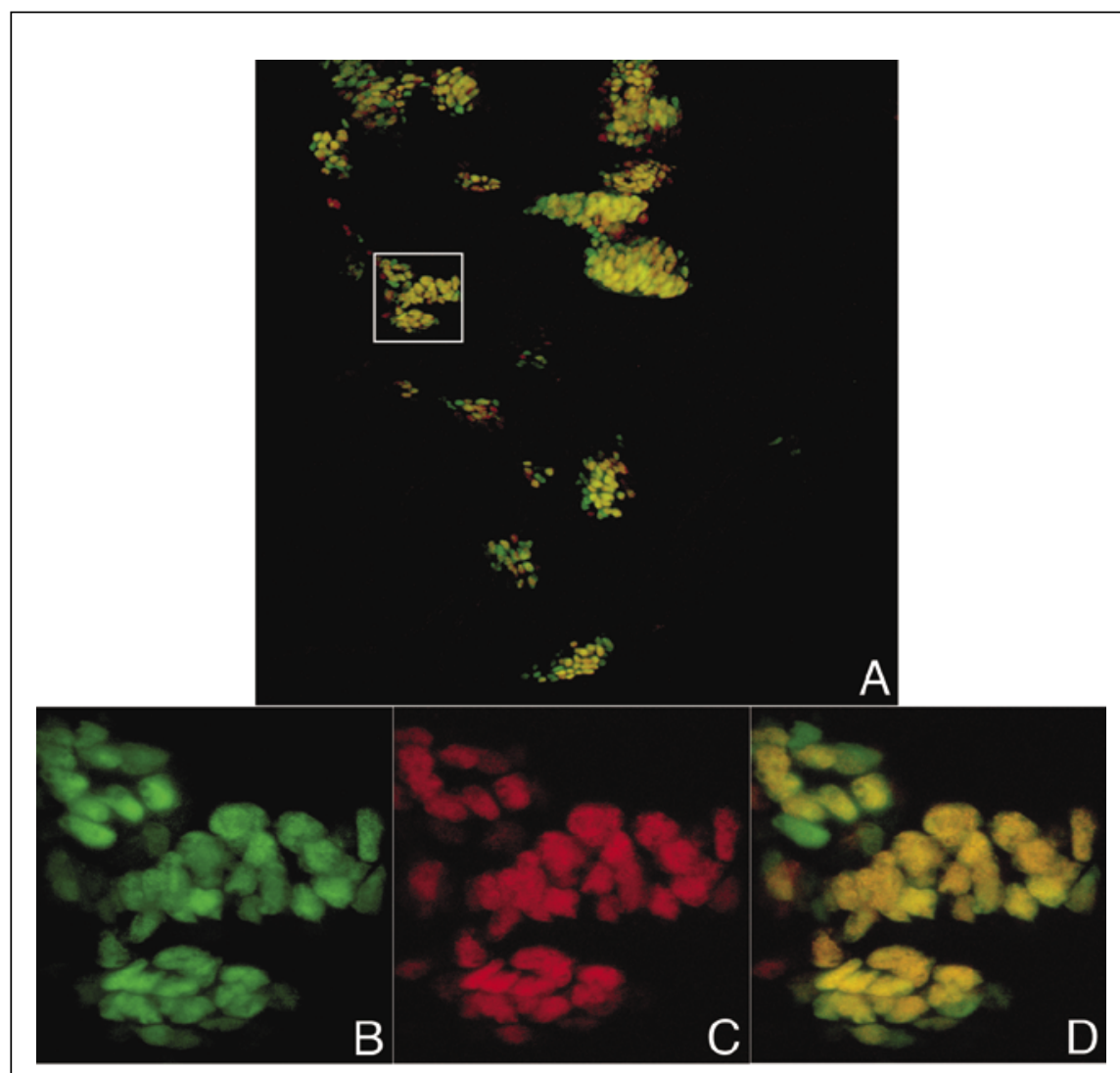

Figure 3. Double labeling of developing "proneural clusters" with EGFP-NLS and DsRed.T4NLS. Shown are confocal images of a single wing imaginal disc from a late third-instar larva carrying one copy each of E(spl)m $\alpha$-EGFP-NLS and E(spl)m $\alpha$-DsRed.T4-NLS. Enhancer of split $m \alpha$ $[E(s p l) m \alpha]$ is expressed specifically in the proneural clusters from which sensory organ precursor cells of the PNS arise $(14,15)$. (A) Low-magnification image of the merged green and red channels, showing nuclei of multiple proneural clusters. Note the prevalence of yellow cells expressing both EGFP-NLS and DsRed.T4-NLS, along with a minority of cells that are predominantly green or red. Boxed region is shown enlarged in panels B-D. (B-D) Higher-magnification images of the green (B), red (C), and merged (D) channels showing the overall coincidence of EGFP-NLS and DsRed.T4-NLS expression and revealing that very few nuclei express exclusively one or the other. EGFP, enhanced green fluorescent protein; NLS, nuclear localization signal.

socket cells remain vital and brightly fluorescent throughout the life of the fly, the expression of the reporter apparently causes a physiological defect in socket cells, resulting in a lack of coordination in adults from most transgenic lines. Socket cells are known to be important for proper mechanoreceptor function and physiology (e.g., see Reference 10). Since the same reporter may be expressed in other cell types with no developmental or behavioral defects (see below) and no such defects have been reported in other cell types or organisms, it would seem that the comparative strength of the ASE together with the polyploidy of the socket cell, the fact that the enhancer is continuously active for most of pupation and all of adult life, and the unique neurophysiological role of the socket cell may be responsible for this defect. Cytoplasmic DsRed.T4 causes milder lack of coordination than DsRed.T4-NLS, perhaps suggesting that large nuclear accumulations of protein are contributing to the behavioral defect. Perhaps additional alterations to DsRed, such as those that result in monomeric forms of the protein (13), can be incorporated into the $\mathrm{T} 4$ variant to eliminate this problem, which in any case appears to be restricted to adult socket cells.

We also examined the expression of DsRed.T4-NLS when driven by a $c i s$-regulatory module from the $E(s p l) m \alpha$ gene, which is expressed in the "proneural clusters" from which sensory organ precursors of the peripheral nervous system arise $(14,15)$. When E(spl)m $\alpha$-EGFP-NLS and E(spl)mo-DsRed.T4-NLS expression are compared directly in the same tissue (the wing imaginal disc of the third-instar larva), there is, overall, a high degree of coincidence (Figure 3 ). Unlike what was observed in the socket cells of ASE-DsRed.T4 pupae, the ratio of GFP to RFP fluorescence is variable from cell to cell, with a small minority of cells exhibiting predominantly red signal, and others exhibiting predominantly green (Figure 3A); however, very few cells display exclusively one or the other (Figure 3, B-D). These red-green differences may reflect small differences in the in vivo maturation times and/or stabilities of EGFP and DsRed.T4 (e.g., see Reference 4). We note also that $\mathrm{E}(\mathrm{spl}) \mathrm{m} \alpha$-DsRed.T4- 
NLS is strongly expressed in embryos in addition to imaginal disc tissue (data not shown).

The similar maturation times of EGFP and DsRed.T4, together with the strong fluorescence of DsRed.T4 and the lack of signal cross-contamination, allow for experiments in which a wild-type enhancer in red can be compared with a mutant form in green, and differences in expression pattern, strength of expression, and temporal patterning changes could be detected. In addition, flow cytometry experiments in which two cell types are purified from the same animal at various developmental stages are also possible with this reagent. Thus, DsRed.T4 would seem to be an excellent tool for in vivo double-label experiments with EGFP. The transformation vectors described herein should facilitate the widespread use of this reporter in Drosophila research.

\section{ACKNOWLEDGMENTS}

We are grateful to B. Glick for his generosity in sharing DsRed mutants. This work was supported by National Institutes of Health grant no. GM46993 to J.W.P.

\section{REFERENCES}

1.Matz, M.V., A.F. Fradkov, Y.A. Labas, A.P. Savitsky, A.G. Zaraisky, M.L. Markelov, and S.A. Lukyanov. 1999. Fluorescent proteins from nonbioluminescent Anthozoa species. Nat. Biotechnol. 17:969-973.

2.Handler, A.M. and R.A. Harrell, II. 2001. Polyubiquitin-regulated DsRed marker for transgenic insects. BioTechniques 31:820828 .

3.Verkhusha, V.V., H. Otsuna, T. Awasaki, H. Oda, S. Tsukita, and K. Ito. 2001. An enhanced mutant of red fluorescent protein DsRed for double labeling and developmental timer of neural fiber bundle formation. J. Biol. Chem. 276:29621-29624.

4.Verkhusha, V.V., I.M. Kuznetsova, O.V.
Stepanenko, A.G. Zaraisky, M.M. Shavlovsky, K.K. Turoverov, and V.N. Uversky. 2003. High stability of Discosoma DsRed as compared to Aequorea EGFP. Biochemistry 42:7879-7884.

5.Baird, G.S., D.A. Zacharias, and R.Y. Tsien. 2000. Biochemistry, mutagenesis, and oligomerization of DsRed, a red fluorescent protein from coral. Proc. Natl. Acad. Sci. USA 97:11984-11989.

6.Bevis, B.J. and B.S. Glick. 2002. Rapidly maturing variants of the Discosoma red fluorescent protein (DsRed). Nat. Biotechnol. 20: 83-87.

7.Barolo, S., L.A. Carver, and J.W. Posakony. 2000. GFP and $\beta$-galactosidase transformation vectors for promoter/enhancer analysis in Drosophila. BioTechniques 29:726-732.

8.Hedley, M.L., H. Amrein, and T. Maniatis. 1995. An amino acid sequence motif sufficient for subnuclear localization of an arginine/serine-rich splicing factor. Proc. Natl. Acad. Sci. USA 92:11524-11528.

9.Phelps, C.B. and A.H. Brand. 1998. Ectopic gene expression in Drosophila using GAL4 system. Methods 14:367-379.

10.Barolo, S., R.G. Walker, A.D. Polyanovsky, G. Freschi, T. Keil, and J.W. Posakony. 2000. A Notch-independent activity of Suppressor of Hairless is required for normal mechanoreceptor physiology. Cell 103: 957-969.

11.Rubin, G.M. and A.C. Spradling. 1982. Genetic transformation of Drosophila with transposable element vectors. Science 218 : 348-353.

12.Lai, E.C., R. Bodner, and J.W. Posakony. 2000. The Enhancer of split Complex of Drosophila includes four Notch-regulated members of the Bearded gene family. Development 127:3441-3455.

13.Campbell, R.E., O. Tour, A.E. Palmer, P.A Steinbach, G.S. Baird, D.A. Zacharias, and R.Y. Tsien. 2002. A monomeric red fluorescent protein. Proc. Natl. Acad. Sci. USA 99: 7877-7882.

14.Lai, E.C., R. Bodner, J. Kavaler, G. Freschi, and J.W. Posakony. 2000. Antagonism of Notch signaling activity by members of a novel protein family encoded by the Bearded and Enhancer of split gene complexes. Development 127:291-306.

15.Wurmbach, E., I. Wech, and A. Preiss. 1999. The Enhancer of split complex of Drosophila melanogaster harbors three classes of Notch responsive genes. Mech. Dev. 80: 171-180.

Received 23 December 2003; accepted 5 February 2004.

Address correspondence to Jim Posakony, Division of Biological Sciences/CDB, University of California San Diego, 9500 Gilman Drive, La Jolla, CA 92093-0349, USA. e-mail:jposakony@ucsd.edu 